\title{
Microfluidic Droplet-Facilitated Hierarchical Assembly for Dual Cargo Loading and Synergistic Delivery
}

\author{
Ziyi Yu, ${ }^{\ddagger}{ }^{\dagger}$ Yu Zheng, ${ }^{\ddagger}{ }^{\S}$ Richard M. Parker, ${ }^{\dagger}$ Yang Lan, ${ }^{\S}$ Yuchao Wu, ${ }^{\S}$ Roger J. Coulston, ${ }^{\S}$ Jing Zhang, \\ Oren A. Scherman, ${ }^{*, \S}$ and Chris Abell ${ }^{*} \dagger$ \\ ${ }^{\dagger}$ Department of Chemistry, University of Cambridge, Lensfield Road, Cambridge CB2 1EW, United Kingdom \\ ${ }^{\S}$ Melville Laboratory for Polymer Synthesis, Department of Chemistry, University of Cambridge, Lensfield Road, Cambridge CB2 \\ 1EW, United Kingdom
}

\section{Supporting Information}

ABSTRACT: Bottom-up hierarchical assembly has emerged as an elaborate and energy-efficient strategy for the fabrication of smart materials. Herein, we present a hierarchical assembly process, whereby linear amphiphilic block copolymers are self-assembled into micelles, which in turn are accommodated at the interface of microfluidic droplets via cucurbit [8] urilmediated host-guest chemistry to form supramolecular microcapsules. The monodisperse microcapsules can be used for simultaneous carriage of both organic (Nile Red) and aqueous-soluble (fluorescein isothiocyanate-dextran) cargo. Furthermore, the well-defined compartmentalized structure benefits from the dynamic nature of the supramolecular interaction and offers synergistic delivery of cargos with triggered release or through photocontrolled porosity. This demonstration of premeditated hierarchical assembly, where interactions from the molecular to microscale are designed, illustrates the power of this route toward accessing the next generation of

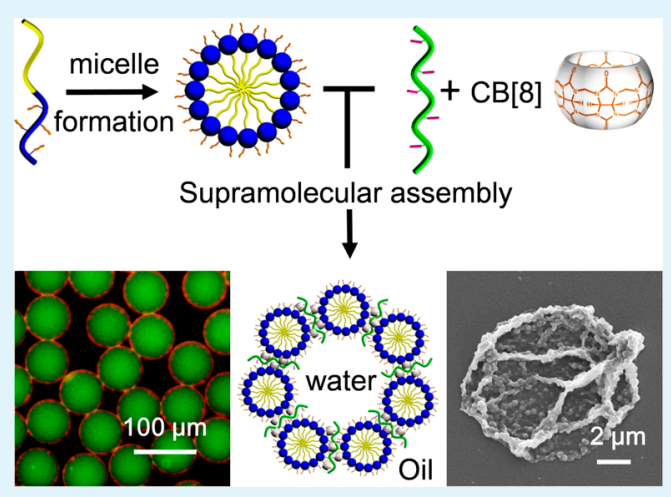
functional materials and encapsulation strategies.

KEYWORDS: hierarchical assembly, microfluidic droplets, cucurbit[8]uril, microcapsules, dual cargo delivery

\section{INTRODUCTION}

Nature assembles diverse structures through self-assembly. ${ }^{1-4}$ This bottom-up approach toward functional structures provides inspiration for the development of the next-generation of advanced smart materials. ${ }^{5-9}$ In the field of polymeric materials, the assembly of block copolymers into defined conformations can mimic the structure of the cell membrane. ${ }^{10-12}$ As an analogy to phospholipids, synthetic amphiphilic block copolymers possess a hydrophilic head and hydrophobic tail, allowing them to self-assemble into multilamellar vesicles and spherical or rod-like micelles. ${ }^{13-16}$ Not only does this assembly process allow investigation of bottom-up assembly, it has generated systems that have potential applications in the fields of material science, bioengineering, and biomedicine. ${ }^{17-21}$ However, due to the lack of target-oriented and predictable assembly protocols, few studies have considered employing block copolymer assemblies as a subunit in the preparation of higher order superstructures. ${ }^{22-24}$ Here, we exploit hierarchical assembly of amphiphilic block copolymers on the molecular, submicro and microscale to form hollow supramolecular microcapsules capable of storage and subsequent controlled release of hydrophobic and hydrophilic incompatible cargos.

Molecular recognition offers the opportunity to guide the assembly of complex structures by controlling the connectivity of the component building blocks. In the supramolecular regime, building blocks are linked by noncovalent interactions, such as hydrogen bonds or hydrophobic forces, to create higher ordered architectures. ${ }^{25-30}$ The reversibility of supramolecular assembly imparts dynamic properties to the resultant material, whereby a specific external stimulus can be used to engineer responsivity, recyclability, self-repairing, and even self-replication. $^{31-35}$

Cucurbit $[8]$ uril $(\mathrm{CB}[8])$ is a barrel-shaped macrocycle that has been extensively applied to the construction of extended supramolecular architectures, exhibiting high mechanical strength, rapid-healing, and stimuli responsiveness. ${ }^{36-39}$ Through the formation of a 1:1:1 ternary host-guest complex, $\mathrm{CB}[8]$ can be viewed as a molecular "handcuff", capable of linking materials with high selectivity and affinity in water $\left(\mathrm{K}_{\text {eq }}\right.$ $\left.\leqslant 10^{12} \mathrm{M}^{-2}\right){ }^{40,41}$ The formation of the ternary complex is stepwise, with initial binding of an electron-deficient aromatic guest (e.g., methyl viologen) necessary for the subsequent inclusion of an electron-rich second guest (e.g., azobenzene). The utility of $\mathrm{CB}[8]$ as a supramolecular cross-linking agent has been illustrated in fabrication of polymeric systems where two complementary polymers are assembled via the host-guest

Received: January 18, 2016

Accepted: March 16, 2016

Published: March 16, 2016 


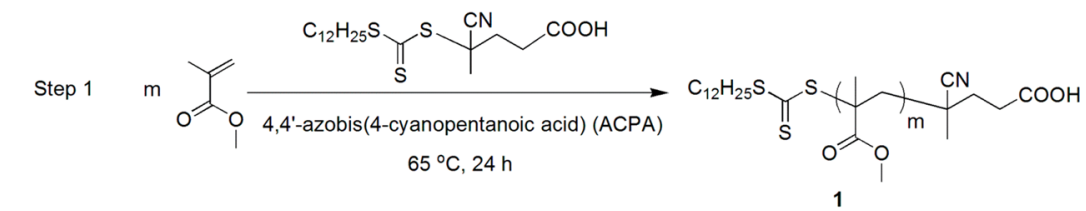

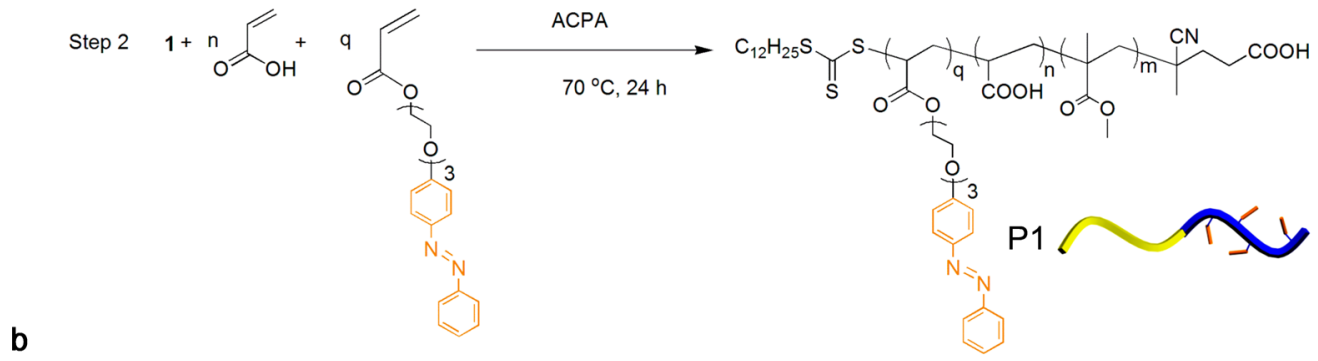

b

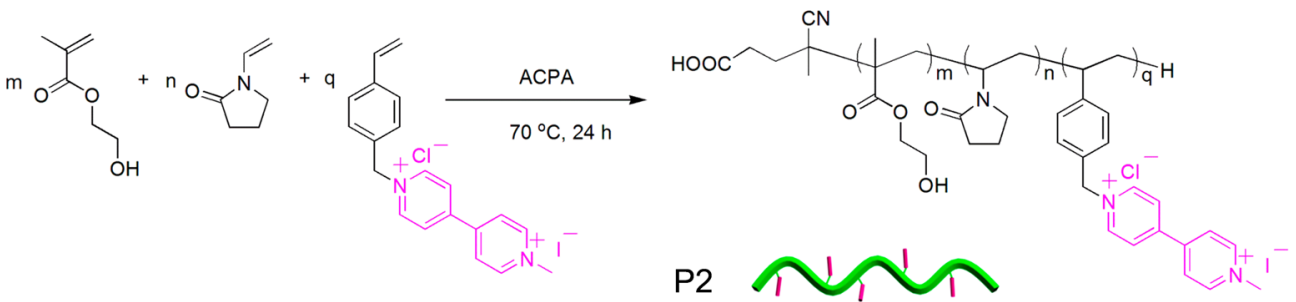

Figure 1. Synthetic scheme for the preparation of (a) azobenzene-functionalized amphiphilic diblock copolymer (P1) and (b) viologenfunctionalized hydrophilic copolymer (P2).

interaction, providing a powerful toolbox for preparation of bespoke materials. $^{42-44}$

Droplet-based microfluidics is a versatile and powerful technology used to generate discrete microdroplets with uniform size and tunable diameter. Each droplet can be considered a microscale version of the traditional chemists' reaction flask, allowing for identical composition and rapid mixing, enabling massively parallelized experimentation. ${ }^{45-47}$ As such, microdroplets offer superb templates to fabricate uniform micromaterials, including hydrogel beads and microcapsules. ${ }^{50-54}$ More recently, "smart" microcapsules containing functional polymers in their membrane have been fabricated from double or triple emulsion microdroplets, enabling control over cargo release via external stimuli. ${ }^{55-58}$ The formation of microcapsules from such nested microdroplets requires either complicated microfluidic devices that control a multistage sequential emulsion or precise control over the wettability of each droplet. ${ }^{59,60}$ Furthermore, the carriage of multiple, incompatible cargos (e.g., hydrophilic/hydrophobic) within a microcapsule relies on compartmentalization between the hollow core and the outer shell, requiring delicate synchronization of the frequencies of microdroplet generation. ${ }^{61-63}$ As such, production of microcapsules with multiple cargos through droplet-based microfluidics remains a significant challenge.

We previously demonstrated a one-step approach to construct supramolecular microcapsules; ${ }^{45,46,53,54}$ these systems (typically) encapsulate hydrophilic cargo(s) within the microcapsule core. The aim here is to utilize this submicron shell as a secondary compartment; enabling synergistic loading of segregated cargos in a prescribed ratio. We have shown that small molecules can be retained within a dendritic microcapsule, ${ }^{47}$ with the caveat that this requires specialized synthesis to prepare the hyper-branched polymers. In contrast, micelles offer ready access to well-defined nanostructures in solution with controllable morphologies, sizes, and functions. ${ }^{48,49}$

In this work, we combine nanoscale micelle formation with $\mathrm{CB}[8]$-mediated molecular-scale recognition within microfluidic droplets to demonstrate the hierarchical assembly of amphiphilic block copolymers, generating supramolecular microcapsules with uniform size and dual-cargo loading. This single emulsion microfluidic approach exploits self-assembly on the molecular, nano- and microscales, whereby block copolymers are assembled into micelles $(\sim 200 \mathrm{~nm})$, which in turn are assembled at the interface of a microdroplet $(\sim 70 \mu \mathrm{m})$ where they are cross-linked via a supramolecular host-guest complex with $\mathrm{CB}[8]$, to form a microcapsule. The segregated domains of the microcapsule core and micellar shell enable simultaneous encapsulation of both hydrophilic and hydrophobic cargos in a single structure. Furthermore, the dynamic nature of the supramolecular cross-links allows for on-demand release of cargo, either by disassembly of the capsule shell or upon a triggered increase in porosity.

\section{RESULTS AND DISCUSSION}

Synthesis of Functional Polymers. The starting amphiphilic diblock copolymer poly(methyl methacrylate)block-poly(acrylic acid) containing a pendant azobenzene (P1) was synthesized via two-step reversible additionfragmentation chain transfer (RAFT) polymerization using the unmodified chain transfer agent 4-cyano-4[(dodecylsulfanylthiocarbonyl)sulfanyl] pentanoic acid (CDTPA) (Figure 1a). CDPTA was used as it successfully mediates the polymerization of a wide variety of monomers in different solvent conditions with control of molecular weight and polydispersity (PDI). The first poly(methyl methacrylate) block 1 was synthesized in 2-butanone using standard RAFT conditions. The feed ratio of methyl methacrylate monomer to 

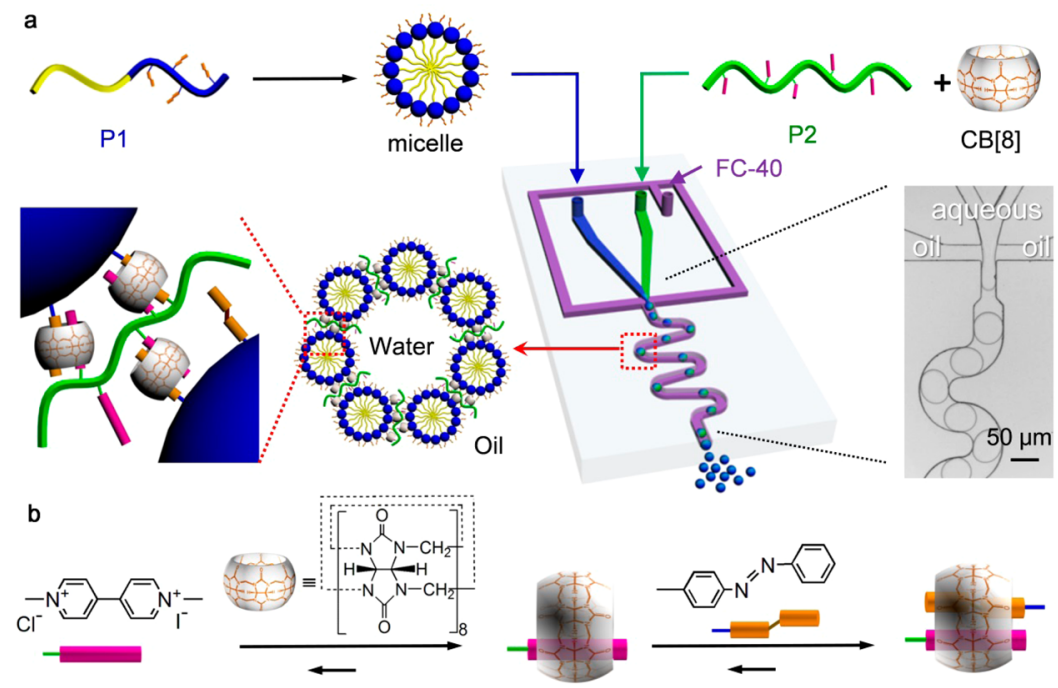

Figure 2. (a) Microdroplet-templated hierarchical assembly of supramolecular microcapsules from amphiphilic blocks copolymers. (b) Step-wise formation of the three-component host-guest complex with $\mathrm{CB}[8]$ in water.
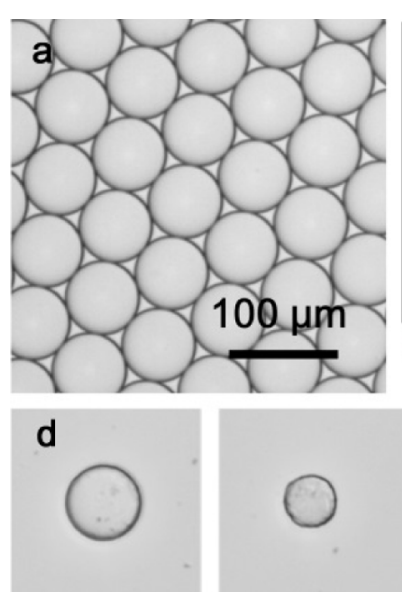

$0 \min$
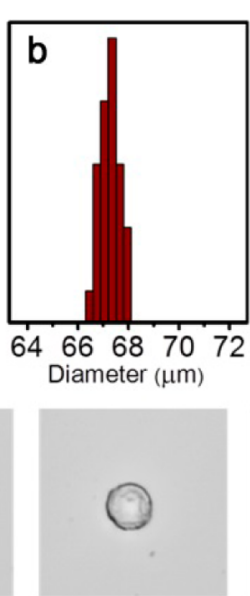

$8 \min$
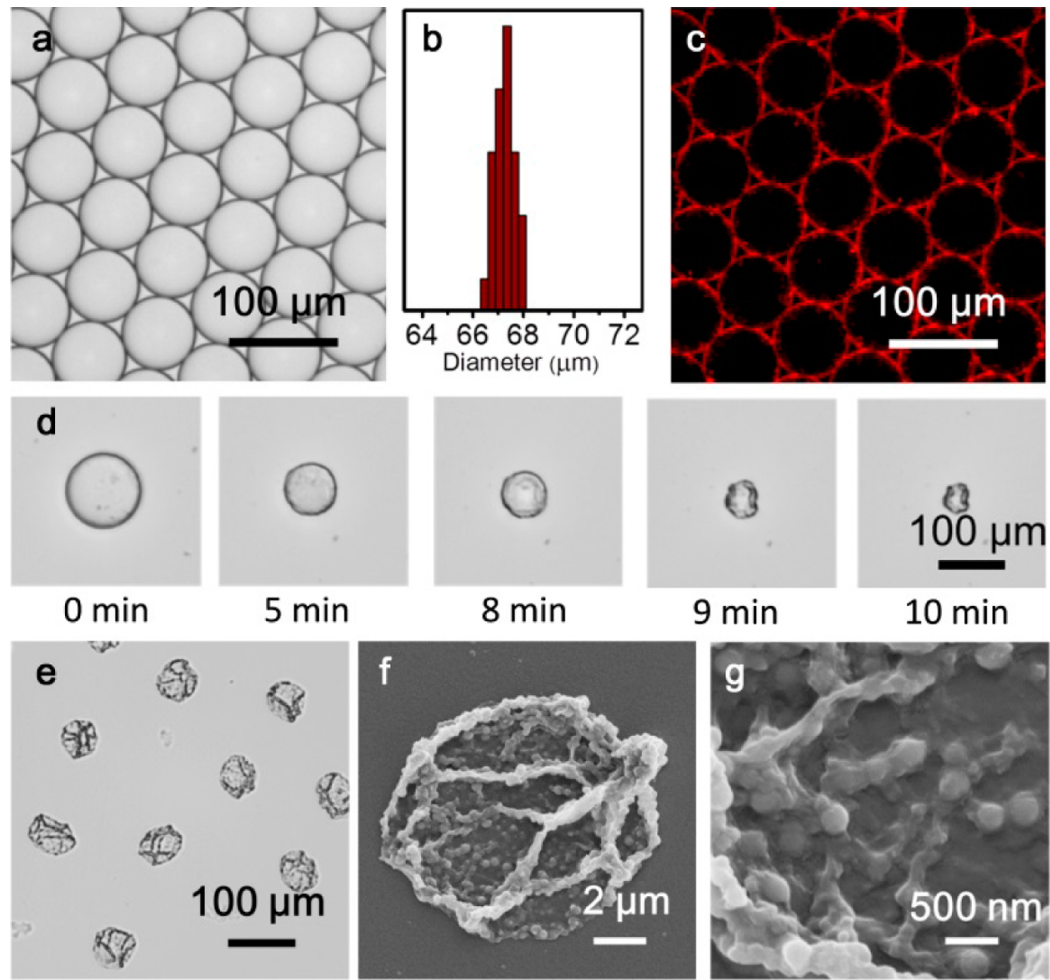

Figure 3. (a) Optical micrograph of aqueous microdroplets containing CB[8], P2 and Nile-Red-loaded P1 micelles. (b) Normalized distribution of microdroplet diameter, exhibiting a narrow size distribution (coefficient of variation $0.6 \%$ ). (c) Fluorescence image of microdroplets, illustrating the assembly of Nile red-loaded $\mathbf{P 1}$ micelles at the droplet interface. (d) Optical micrographs of evaporative microcapsule formation, exemplifying the collapsing structure. (e) Optical micrograph and ( $\mathrm{f}, \mathrm{g}$ ) scanning electron microscope (SEM) images of dry microcapsules, illustrating the micelles embedded within the skin.

CDPTA was 50:1. Analysis by ${ }^{1} \mathrm{H}$ NMR spectroscopy showed the polymerization reached $83 \%$ conversion after $24 \mathrm{~h}$. Tetrahydrofuran (THF) gel permeation chromatography (GPC) analysis reported the average molecular weight $\left(M_{\mathrm{w}}\right)$ of the poly(methyl methacrylate) block as $4.2 \mathrm{kDa}(\mathrm{PDI}=1.1)$, indicating good control over the extent of polymerization. The formed poly(methyl methacrylate) polymer then underwent a second block chain extension polymerization by adding initiator, acrylic acid monomer and azobenzene acrylate monomer. The GPC showed that the $M_{\mathrm{w}}$ increased from 4.2 $\mathrm{kDa}$ to $16.0 \mathrm{kDa}$ after chain extension. ${ }^{1} \mathrm{H}$ NMR analysis showed the diblock copolymer structure contained $4.5 \mathrm{~mol} \%$ azobenzene guests in the final polymer, P1 (Figure S1, Supporting Information).

To synthesize a methyl viologen (MV) multivalent side-chain functional polymer, we used one-step conventional free radical polymerization to prepare water-soluble poly $(N$-vinylpyrrolidone)-co-poly(hydroxyethyl methacrylate)-co-poly(MV-styr- 

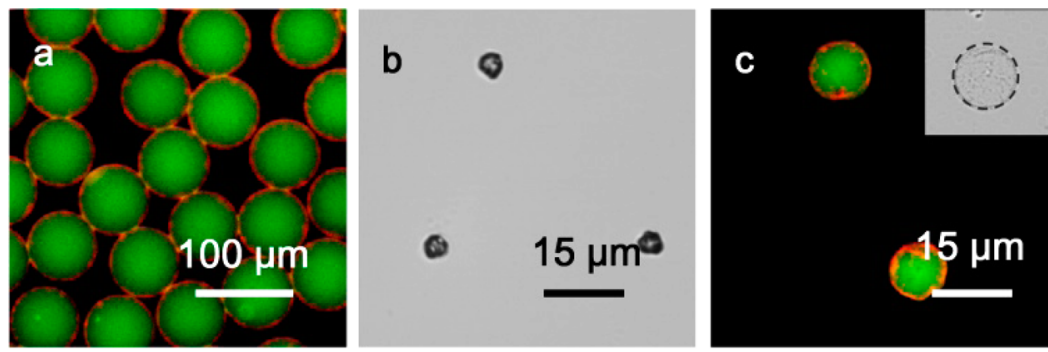

Figure 4. (a) Fluorescent image of monodisperse microcapsules containing both water-soluble FITC-dextran cargo (500 kDa, green) and the hydrophobic dye, Nile Red. (b) Optical micrograph of dry microcapsules and (c) fluorescent micrograph of microcapsules after rehydration.

ene) (P2, Figure 1b). A styrenic monomer bearing a MV moiety was synthesized from 4-vinylbenzyl chloride and methyl bipyridinium iodide salt in high yield (85\%). Water-soluble monomer $\mathrm{N}$-vinylpyrrolidone and hydroxyethyl methacrylate were used to copolymerize with styrenics to form the copolymer, P2. Aqueous GPC and ${ }^{1} \mathrm{H}$ NMR demonstrated that P2 had $M_{\mathrm{w}}$ of $27 \mathrm{kDa}(\mathrm{PDI}=1.4)$ and contained approximately of $8.3 \mathrm{~mol} \%$ of $\mathrm{MV}$ guest. (Figure S2, Supporting Information).

Microfluidic Droplet-Facilitated Hierarchical Assembly. The hierarchical assembly process, whereby linear amphiphilic block copolymers are self-assembled into micelles, which in turn are assembled at the interface of monodisperse microfluidic droplets to form supramolecular microcapsules, is illustrated in Figure 2. The first stage of assembly occurs through the micellization of amphiphilic diblock copolymer P1 (Figure 2a). In aqueous media, P1 directs the hydrophilic poly(acrylic acid) head into the solvent, while shielding the hydrophobic poly(methyl methacrylate) tail to form micelles. As a result, the hydrophobic dye, Nile Red, is encased within the hydrophobic core of $\mathbf{P 1}$ micelles, enabling it to be dispersed in water (Figure S3, Supporting Information). The peak of the emission spectrum of Nile Red shifted from 592 to $620 \mathrm{~nm}$ when the Nile Red was transferred from tetrahydrofuran to water in micelles (Figure S4, Supporting Information). ${ }^{64,65}$ More importantly, the outer face of the micelle can be selectively functionalized. Here, the presence of pendant azobenzene groups within the hydrophilic head of the micelles facilitates supramolecular assembly via $\mathrm{CB}[8]$ (Figure 2b), allowing them to be employed as subunits in the construction of higher level structures.

The second stage of hierarchical assembly occurs from $\mathrm{CB}[8]$-mediated host-guest interaction, whereby $\mathbf{P 1}$ micelles are assembled at the interface of microfluidic droplets via an electrostatic interaction with a charged-surfactant. ${ }^{53}$ As shown in Figure 2a, an aqueous flow containing $\mathbf{P 2}$ and $\mathrm{CB}[8]$ was coinjected with a second flow containing Nile-Red-loaded P1 micelles into a microfluidic flow-focusing device. After injection, the two fluids met as a laminar coflow, before arrival at the flow-focusing junction where it was segmented into microdroplets by a perpendicular flow of perfluorinated oil. After generation, the spherical microdroplets passed through a winding channel to encourage thorough mixing of the three components, before collection for further study.

Aqueous microdroplets containing Nile Red-loaded P1 micelles, P2, and $\mathrm{CB}[8]$ were generated at a combined aqueous flow rate of $100 \mu \mathrm{L} / \mathrm{h}$, with the continuous oil phase (Fluorinert FC-40, 3 wt \% fluorous surfactant "XL-01-171" and 1.5 wt \% charged-surfactant "amine-Krytox") injected at 200 $\mu \mathrm{L} / \mathrm{h}$. The equimolar concentration of MV: azobenzene: $\mathrm{CB}[8]$ within the mixed microdroplet was $15 \mu \mathrm{M}$, allowing for formation of the 1:1:1 heteroternary complex. As shown in Figure 3a, at this flow rate the microdroplets exhibit a low level of polydispersity, with a mean diameter of $67 \mu \mathrm{m}$ and coefficient of variation of $0.6 \%$ (Figure $3 \mathrm{~b}$ ). Fluorescence microscopy was used to track the assembly within the microdroplets; as shown in Figure 3c, fluorescence from Nile Red is localized at the water/oil interface, illustrating the formation of the supramolecular microcapsule. On evaporation of the aqueous microdroplet at room temperature, the interfacial assembly of $\mathbf{P 1}$ micelles is locked by $\mathbf{P 2}$, via supramolecular $\mathrm{CB}[8]$ cross-links, to give hollow microcapsule shells.

In contrast to solid microspheres, the prepared microcapsules comprise an empty core and a supramolecular composite shell. As a result, evaporation of the aqueous microdroplet led to a steady decrease in diameter until cross-linking density at the interface was sufficient for an elastic shell to form. After this point, further evaporation resulted in collapse and distortion of this flexible shell, until eventual collapse onto the glass substrate (Figure 3d). As shown in Figure $3 \mathrm{e}$, isolated stable microcapsules with uniform size remain after dehydration of the microdroplets. To assess the substructure of the microcapsule shell, the surface morphology was studied by scanning electron microscopy (SEM). Figure $3 \mathrm{f}$ reveals that microcapsules collapse upon drying due to a lack of internal support, with folds and creases clearly visible on the surface. The enlarged image (Figure $3 \mathrm{~g}$ ) further shows that the microcapsule skin consists of a composite network of micelles embedded within a matrix of $\mathbf{P 2}$.

The hierarchical microcapsule structure allows for both hydrophilic and hydrophobic cargos to be loaded simultaneously. To illustrate this concept, microcapsules were prepared that encapsulated both the water-soluble cargo, fluorescein isothiocyanate-dextran (FITC-dextran, $500 \mathrm{kDa}$ ) and the organic-soluble dye, Nile Red. As illustrated in Figure 4a, FITC-dextran (green fluorescence) was loaded within the aqueous flow and correspondingly is located within the core of the microcapsule, whereas Nile Red is trapped within the micelles that comprise the microcapsule shell (red fluorescence). The cargo-loaded microcapsules were collected and airdried, forming stable dehydrated structures that can be stored and rehydrated (Figure $4 \mathrm{~b}, \mathrm{c}$ ). When loaded with cargo, osmotic pressure results in a near-doubling of the dry microcapsule diameter during rehydration; however, the supramolecular cross-link is still sufficient to retain both encapsulated cargos without leakage (Figure 4c). Furthermore, the microcapsules were shown to survive five cycles of dehydration/rehydration, with the integrity of the microcapsule 


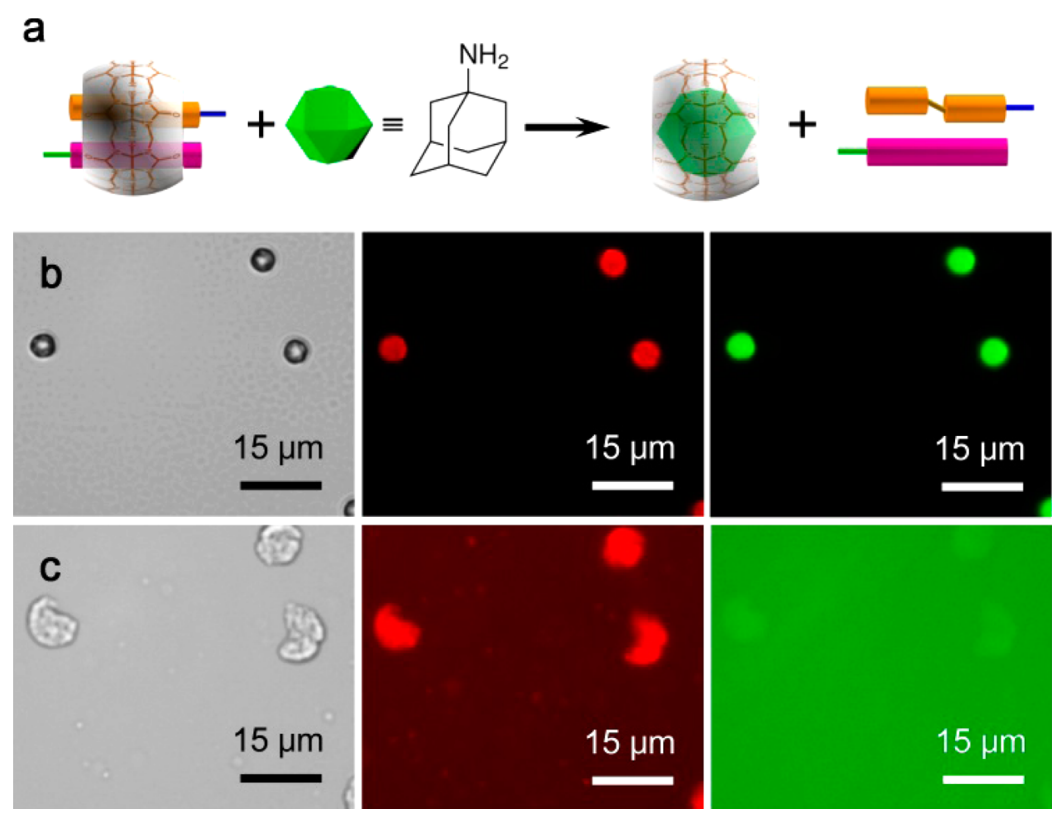

Figure 5. (a) Schematic of the disassembly of the ternary complex between MV, azobenzene, and $\mathrm{CB}[8]$, in the presence of competitive guest, 1adamantylamine (ADA). Optical and fluorescent micrographs of dual cargo-loaded (Nile Red and 500 kDa FITC-dextran) microcapsules; (b) before and (c) after hydration for $3 \mathrm{~min}$ in an aqueous solution of 1-adamantylamine $(1 \mathrm{mM})$.
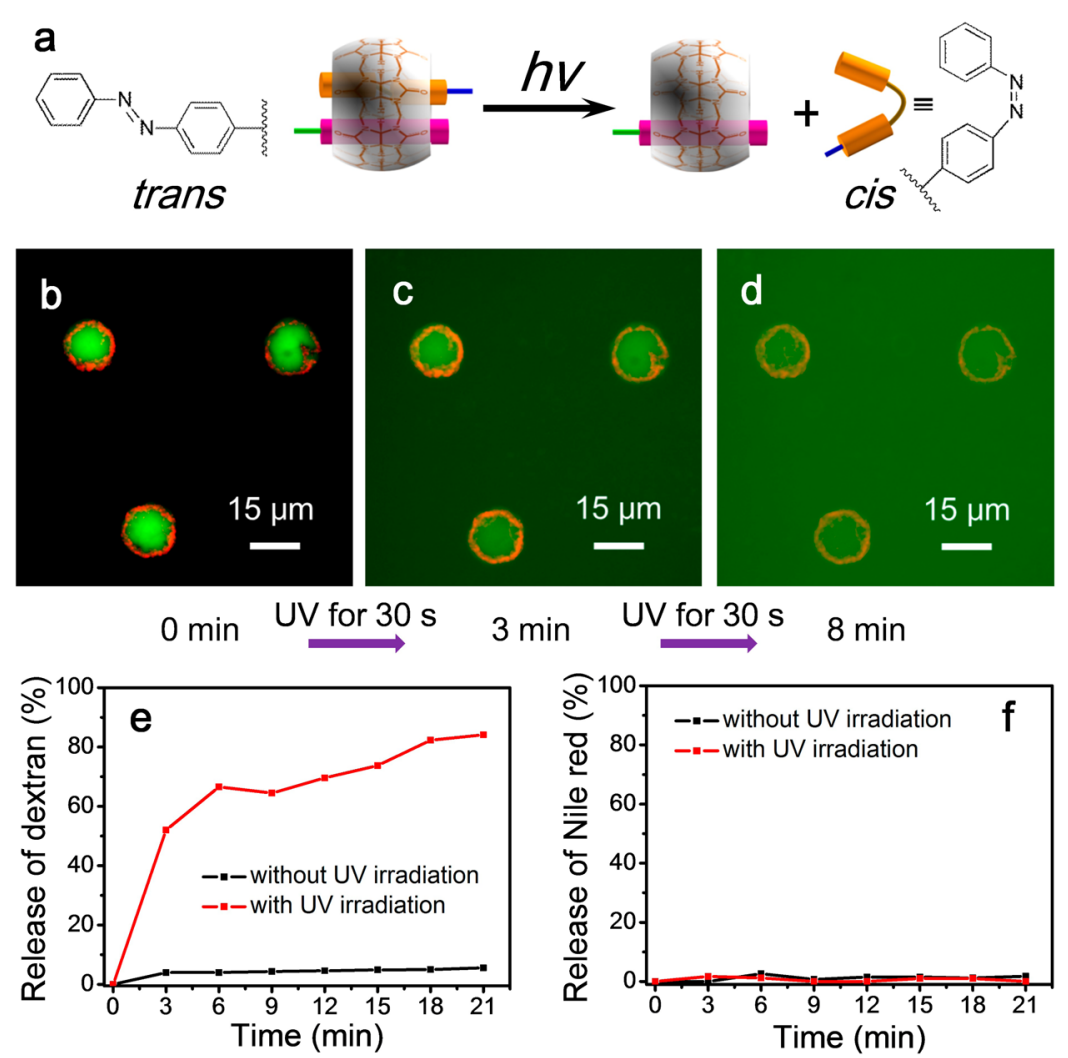

Figure 6. (a) Schematic of the photochemical disassembly of the ternary complex between MV, azobenzene, and CB[8]. (b-d) Fluorescent micrographs of the triggered release of $500 \mathrm{kDa}$ FITC-dextran cargo from hydrated microcapsules, upon exposure to ultraviolet light. Release profiles of (e) FITC-dextran and (f) Nile Red from microcapsules as a function of the rehydration time.

skin evidenced by the retention of the encapsulated cargo (Figure S5, Supporting Information).

The efficacy of the supramolecular microcapsules for the storage and release of cargo was investigated using a family of FITC-dextran as molecular probes. After rehydration for 30 min, $500 \mathrm{kDa}$ FITC-dextran was fully retained by the microcapsule, whereas $250 \mathrm{kDa}$ FITC-dextran displayed weak permeability and $70 \mathrm{kDa}$ was able to diffuse freely into the external environment (Figure S6, Supporting Information). 500 $\mathrm{kDa}$ FITC-dextran has a Stoke's radius of around $14.7 \mathrm{~nm}$, ${ }^{66}$ given that it is not able to readily diffuse out of the 
microcapsules this data suggests an upper limit for the micropore size of the capsule membrane.

Disassembly and Controlled Release of Cargo. The noncovalent nature of supramolecular interactions offers an opportunity to disassemble the microcapsule shell in a controlled manner, and correspondingly release encapsulated cargo. The introduction of a competitive guest for $\mathrm{CB}[8]$, such as 1-adamantylamine (ADA), is one such approach (Figure 5a). Cargo-loaded microcapsules were prepared as above, but instead rehydrated in an aqueous solution of $\mathrm{ADA}(1 \mathrm{mM})$. As shown in Figure $5 b, c$, immediate disassembly of the microcapsules was observed, with triggered dispersal of both hydrophilic FITC-dextran and hydrophobic Nile Red cargo (within micelles) into the bulk media. The released cargo was also examined by fluorescence spectroscopy (Figure S7, Supporting Information), which shows two fluorescence emission peaks of $520 \mathrm{~nm}$ (released FITC-dextran cargo) and $617 \mathrm{~nm}$ (released Nile Red cargo) upon ADA triggering.

Photochemistry offers a route to control the extent of supramolecular cross-linking. The azobenzene moiety can undergo a reversible trans-to-cis photoisomerization under exposure to UV light (Figure 6a). This leads to dissociation of the 1:1:1 heteroternary complex to give a MVCCB[8] binary complex and free cis-azobenzene. Photoisomerization of the azobenzene does not lead to quantitative disassembly, with the photostationary state reported to be up to $80 \%$ cis- in the presence of $\mathrm{CB}[8] .{ }^{67}$ To investigate the suitability of photochemical stimulation for controlled, sustained release of cargo, we hydrated microcapsules loaded with FITC-dextran $(500 \mathrm{kDa})$ in water and then exposed to ultraviolet light $(30 \mathrm{~s}$, $\lambda_{\max }=377 \mathrm{~nm}$ ). As shown in Figure $6 \mathrm{~b}-\mathrm{d}$ the photoinduced reduction in cross-linking allowed the microcapsule to enlarge, resulting in release of the previously retained FITC-dextran cargo. A control experiment in the absence of UV light did not show any release of FITC-dextran cargo (Figure S8, Supporting Information).

The release of both cargoes was quantified using a fluorescence spectrometer, given that there was no significant photobleaching of either FITC-dextran or Nile Red under 3.5 min UV irradiation from a LZC-ORG photoreactor $\left(\lambda_{\max }=377\right.$ $\mathrm{nm}$, Figure S9, Supporting Information). As shown in Figure 6e, exposure to UV for $3 \mathrm{~min}$ led to an increase in the porosity of the microcapsule that gave rise to the release of FITCdextran cargo; with $52 \%$ released after 3 min irradiation, increasing to $84 \%$ after a further $18 \mathrm{~min}$ in the dark. A control experiment showed that in the absence of any UV exposure, only $6 \%$ of FITC-dextran cargo was released over this timescale. In contrast to FITC-dextran, a negligible release of Nile Red in micelles was observed after 21 min rehydration, either with or without exposure of the microcapsules to UV light (Figure 6f, Figure S10 in Supporting Information). This differs dramatically from $\mathrm{ADA}$-triggered release, where both cargoes were rapidly dispersed into the surrounding media (Figure 5). Such differing release profiles is attributed to incomplete photoisomerisation of the azobenzene units to the cis-isomer upon exposure to UV light, resulting in a reduction in the number of cross-links within the polymer shell, but not complete disassembly as seen with ADA. This has the effect of increasing the porosity of the capsule shell and thus enables release of cargo from the core, but does not break apart the capsule resulting in retention of cargo in the shell. The ability to tune the porosity of the microcapsule without disassembling the shell allows for the exclusive or stepwise release of only the macromolecular cargo, with the hydrophobic small molecule cargo Nile Red fully retained (Figure $6 f$ and Figure S10, Supporting Information). This phenomenon could be employed in the future to construct a biphasic microreactor, whereby hydrophobic catalysts are immobilized in the shell of the microcapsule, with flow in and out of hydrophilic reagents and products, respectively, controlled remotely by photochemical triggers.

\section{CONCLUSIONS}

In summary, by combining nanoscale micellization with $\mathrm{CB}[8]$ mediated molecular-scale recognition in micron-scale droplets, we have demonstrated the hierarchical assembly of amphiphilic block copolymers to form supramolecular microcapsules. The resultant microcapsules are monodisperse and offer good multiencapsulation efficiency with synergistic release via the supramolecular cross-links. The hierarchical structure further allows for both hydrophilic and hydrophobic domains, enabling simultaneous carriage of incompatible cargos with Nile Red and FITC-dextran. Moreover, we demonstrate that the $\mathrm{CB}[8]$ supramolecular cross-link can be used to both disassemble the microcapsule under mild conditions (upon additional of a competitive guest) and to tune the properties of the microcapsule skin with UV-controlled porosity.

The high degree of customization enabled by our bottom-up hierarchical approach, combined with the simplicity of microdroplet preparation make this a powerful and flexible system with many applications, for example, in homecare and cosmetic products, and the light-controlled cargo release behavior can be potentially applied to delivery UV-resistant compounds in skincare products. The hierarchical assembly in this study is focused on the design and proof-of-concept application of the fabrication method and the triggering mechanism, but we anticipate further development of the $\mathrm{CB}[8]$ supramolecular system in droplet-based microfludics will rapidly lead to exploitable applications.

\section{EXPERIMENTAL SECTION}

Materials. Methyl methacrylate, hydroxyethyl methacrylate, $\mathrm{N}$ vinylpyrrolidone and acrylic acid monomers were purchased from Sigma-Aldrich and were passed through a column of silica gel and purged with high purity nitrogen for $1 \mathrm{~h}$ prior to use. Nile Red (analytical standard), 4-cyano-4-[(dodecylsulfanylthiocarbonyl)sulfanyl]pentanoic acid, and 2-butanone (HPLC grade), fluorescein isothiocyanate-labeled dextran (FITC-dextran), and 1-adamantylamine (ADA) were purchased from Sigma-Aldrich. Styrenic monomer bearing methyl viologen moieties (MV-styrene), azobenzene acrylate monomer and cucubit [8] uril $(\mathrm{CB}[8])$ were synthesized as previously reported. $^{68,69}$ Solvents and reagents were used without further purification unless otherwise stated. All aqueous solutions were made in deionized water treated with a Milli- $\mathrm{Q}$ reagent system with a resistivity of $18.2 \mathrm{M} \Omega / \mathrm{cm}$ at $25{ }^{\circ} \mathrm{C}$.

Characterization. ${ }^{1} \mathrm{H}$ NMR spectra $(400 \mathrm{MHz})$ were recorded using a Bruker Avance QNP 400 Ultrashield spectrometer, equipped with a $5 \mathrm{~mm}$ BBO ATM probe with a $z$-gradient. Chemical shifts are recorded in $\mathrm{ppm}(\delta)$ in $\mathrm{D}_{2} \mathrm{O}$ with the internal reference set to 4.67 . Weight-average molecular weight $\left(M_{\mathrm{w}}\right)$, number-average molecular weight $\left(M_{\mathrm{n}}\right)$ and polydispersity $\left(M_{\mathrm{w}} / M_{\mathrm{n}}\right)$ were obtained by aqueous or tetrahydrofuran (THF) GPC. The aqueous GPC setup consisted of a Shodex OHpak SB column, connected in series with a Shimadzu SPDM20A prominence diode array detector, a Wyatt DAWN HELEOS multiangle light scattering detector and a Wyatt Optilab rEX refractive index detector. The THF GPC setup consisted of two $30 \mathrm{~cm}$ PLgel Mixed-C columns in series, eluted using THF and calibrated against a series of 12 near-monodisperse poly(methyl methacrylate) standards 
(Mp from 690 to $1944000 \mathrm{~g} \cdot \mathrm{mol}^{-1}$ ). The polymers were analyzed in THF at a concentration of $5.0 \mathrm{mg} / \mathrm{mL}$. All calibrations and analysis were performed at $35{ }^{\circ} \mathrm{C}$ and a flow rate of $1 \mathrm{~mL} / \mathrm{min}$. Photoluminescence (PL) emission spectra of FITC-dextran and Nile Red were obtained using a Varian Cary Eclipse fluorescence spectrophotometer. Transmission electron microscopy (TEM) characterization was carried out by a FEI Philips Tecnai 20 TEM under an accelerating voltage of $80 \mathrm{kV}$. Size distribution of P1 micelles were performed by dynamic light scattering (DLS) with Malvern Zeta sizer NS90 instrument. Images of microdroplets formation were obtained using a Phantom v7.2 camera attached to an Olympus IX71 inverted microscope. Microscopic images and fluorescence images were obtained using an Olympus IX81 inverted optical microscope coupled with a camera of Andor Technology EMCCD iXonEM+ DU 897. To image the fluorescence of FITC-dextran, a mercury lamp was installed for wide-spectrum illumination with "FITC" filters and dichroics fitted to separate the fluorescence excitation and emission light. Scanning electron microscopy (SEM) measurements were made and images recorded using a Leo 1530 variable pressure SEM with InLens detector.

Synthesis of Poly(methyl methacrylate)-block-poly(acrylic acid) Containing Azobenzene Pendant (P1). Amphiphilic diblock polymer poly(methyl methacrylate)-block-poly(acrylic acid) containing azobenzene guest was synthesized via two-step reversible additionfragmentation chain transfer (RAFT) polymerization using the unmodified chain transfer agent 4-cyano-4-[(dodecylsulfanylthiocarbonyl)sulfanyl]pentanoic acid (CDTPA).

Step 1: To a two-necked round-bottom flask were added CPTPA (201 mg, $0.5 \mathrm{mmol}, 1$ equiv) and methyl methacrylate (2.5 g, 25 mmol, 50 equiv) in butanone $(8 \mathrm{~mL})$. Oxygen was removed by bubbling argon through the solution for $20 \mathrm{~min}$, followed by the subsequent addition of 4,4-azobis(4-cyanovaleric acid) (ACPA, $21 \mathrm{mg}$, $0.075 \mathrm{mmol}, 0.15$ equiv). The flask was then immersed in a preheated oil bath $\left(65^{\circ} \mathrm{C}\right)$ and the solution stirred at $400 \mathrm{rpm}$ for $24 \mathrm{~h}$. The resultant polymer was precipitated into cold hexane and dried under vacuum. The formed polymer was characterized to give $M_{\mathrm{w}}=4150 \mathrm{Da}$, PDI $=1.1$ from GPC, methyl methacrylate conversion $=83 \%$ from ${ }^{1} \mathrm{H}$ NMR spectroscopy.

Step 2: To a two-necked round-bottom flask were added poly(methyl methacrylate) from step $1(400 \mathrm{mg}, 0.1 \mathrm{mmol}, 1$ equiv), acrylic acid (720 mg, $10 \mathrm{mmol}, 100$ equiv), and azobenzene acrylate monomer ( $200 \mathrm{mg}, 0.5 \mathrm{mmol}, 5$ equiv) in butanone $(4 \mathrm{~mL})$. Oxygen was removed by bubbling argon through the solution for 20 min, followed by the subsequent addition of ACPA ( $7 \mathrm{mg}, 0.025$ mmol, 0.25 equiv). The flask was then immersed in a preheated oil bath $\left(70{ }^{\circ} \mathrm{C}\right)$ and the solution stirred at $400 \mathrm{rpm}$ for $24 \mathrm{~h}$. The resultant polymer was precipitated into cold hexane and dried under vacuum. The formed polymer was characterized to give $M_{\mathrm{w}}=16 \mathrm{kDa}$, $\mathrm{PDI}=1.3$ from tetrahydrofuran (THF) GPC, [methyl methacrylate][acrylic acid]-[azobenzene] = 40:62:4.8 from ${ }^{1} \mathrm{H}$ NMR (Figure S1, Supporting Information).

Synthesis of Poly(N-vinylpyrrolidone)-co-poly(hydroxyethyl methacrylate)-co-poly(MV-styrene) (P2). Poly( $N$-vinylpyrrolidone)-co-poly(hydroxyethyl methacrylate)-co-poly(MV-styrene) was synthesized via free radical polymerization. To a two-necked roundbottom flask were added N-vinylpyrrolidone $(450 \mathrm{mg}, 4 \mathrm{mmol}, 80$ equiv), hydroxyethyl methacrylate ( $2.6 \mathrm{~g}, 20 \mathrm{mmol}, 400$ equiv) and MV styrene monomer ( $0.9 \mathrm{~g}, 2 \mathrm{mmol}, 40$ equiv) in methanol/water $(\mathrm{v} / \mathrm{v}=50: 50,10 \mathrm{~mL})$. Oxygen was removed by bubbling argon through the solutions for $20 \mathrm{~min}$, followed by the subsequent addition of ACPA ( $14 \mathrm{mg}, 0.05 \mathrm{mmol}, 1$ equiv). The flask was immersed in a preheated oil bath $\left(70^{\circ} \mathrm{C}\right)$ and the solution stirred at $400 \mathrm{rpm}$ for 24 $\mathrm{h}$. The polymer was precipitated into cold hexane and finally dried in vacuo at $50{ }^{\circ} \mathrm{C}$. The resultant polymer was dialyzed in water through a MWCO 6000-8000 membrane and freeze-dried. The formed polymer was characterized to give $M_{\mathrm{w}}=27 \mathrm{kDa}, \mathrm{PDI}=1.4$ from aqueous GPC, [hydroxyethyl methacrylate $]-[\mathrm{N}$-vinylpyrrolidone $]-[\mathrm{MV}]=$ 100:22:11 from ${ }^{1} \mathrm{H}$ NMR spectroscopy (Figure S2, Supporting Information).
Self-Assembly of P1 into Micelles. A typical procedure for the preparation of micelles dispersions is as follows: $4 \mathrm{mg}$ of amphiphilic diblock copolymer P1 was dissolved in $3 \mathrm{~mL}$ of THF. Under vigorous stirring, $10 \mathrm{~mL}$ of deionized water was then slowly added. After the addition was complete, the micellar solution was further stirred for 24 $\mathrm{h}$, upon which the THF had fully evaporated.

To form Nile-Red-loaded P1 micelles, first $0.5 \mathrm{~mL}$ of Nile Red solution $\left(1.5 \times 10^{-3} \mathrm{mg} / \mathrm{mL}\right.$ in THF) was added to $3 \mathrm{~mL} \mathrm{THF}$ containing amphiphilic diblock copolymer P1 $(4 \mathrm{mg})$. Under vigorous stirring, $10 \mathrm{~mL}$ of deionized water was then slowly added. After the addition was complete, the micellar solution was further stirred for 24 $h$, upon which the THF had fully evaporated.

Supramolecular-Assembly of Micelles in Microfluidic Droplets. The flow-focusing poly(dimethylsiloxane) microfluidic device was produced via soft lithography as previously described, ${ }^{52}$ with three inlets and one outlet. The depth of microfluidic channel was $50 \mu \mathrm{m}$ and the nozzle was $40 \mu \mathrm{m}$ wide. To generate water-in-oil microdroplets, three different liquids were injected into the three inlets, by three syringe pumps (PHD, Harvard Apparatus) with controlled flow rates. Flourinert FC-40 (3M) containing a 3 wt \% fluorous surfactant (XL-01-171, Sphere Fluidics Ltd.) and 1.5 wt \% amine-Krytox $^{53}$ (Sphere Fluidics Ltd.) was used as the continuous phase. The first discontinuous aqueous phase comprised P1 micelles dispersed in water, and the second discontinuous phase was a solution of $\mathbf{P 2}$ and $\mathrm{CB}[8]$. The continuous phase and both discontinuous phases were loaded into three $1 \mathrm{~mL}$ syringes, before connecting to the microfluidic chip. Syringes with needles were mounted on syringe pumps and fitted with polyethylene tubing, while the other end of the tubing was inserted into the appropriate inlets of a microfluidic chip. Microdroplets formation was initiated as Flourinert FC-40 was first pumped into the device at the rate of $200 \mu \mathrm{L} / \mathrm{h}$ to fill the appropriate channels. Each aqueous dispersed phase was then pumped into the device at $50 \mu \mathrm{L} / \mathrm{h}$. In a typical experiment, the final concentration of $\mathrm{MV}$, azobenzene, and $\mathrm{CB}[8]$ was $15 \mu \mathrm{M}$. After formation, microdroplets were either collected in a PDMS reservoir downstream or transferred to a glass bottom dish. Upon collection, droplets were allowed to dehydrate over $5 \mathrm{~h}$ for the complete formation of isolated microcapsules. After drying on a glass slide, the prepared microcapsules were washed with Novec HFE-7100 (3M) three times to remove the residual surfactants.

To generate FITC-dextran loaded microcapsules, FITC-dextran $(500 \mathrm{kDa}, 250 \mathrm{kDa}$, or $150 \mathrm{kDa})$ was directly mixed with the aqueous $\mathbf{P 2}$ and $\mathrm{CB}[8]$ solution and then pumped into the microfluidic device as above. The final concentration of FITC-dextran within the microdroplet was $0.25 \mathrm{mg} / \mathrm{mL}$.

Rehydration of Supramolecular Microcapsules and Subsequent Release of Cargo. To rehydrate the FITC-dextran loaded hierarchical microcapsules, the glass slide was mounted on to a fluorescence microscope (Olympus IX81) and $50 \mu \mathrm{L}$ of water added to the microcapsule-coated region. The 5 cycles of dehydration/ rehydration were tested with a sample of $500 \mathrm{kDa}$ FITC-dextran loaded microcapsules and the period of each cycle was $1 \mathrm{~h}$.

The disassembly of FITC-dextran/Nile Red dual-loaded hierarchical microcapsules and their triggered release behavior were tested using an aqueous solution of $\mathrm{ADA}(50 \mu \mathrm{L}, 1 \mathrm{mM})$. The $\mathrm{ADA}$ solution was applied over the dried microcapsules and optical and fluorescence images were collected after $3 \mathrm{~min}$ rehydration. Optical and fluorescent images were taken by Olympus IX81 microscope under 40× objective.

To investigate photochemical disassembly, optical and fluorescent images were taken by Olympus IX81 microscope under 40× objective. $500 \mathrm{kDa}$ FITC-dextran loaded microcapsules were rehydrated in water and then exposed to a focused UV light beam $\left(30 \mathrm{~s}, \lambda_{\max }=377 \mathrm{~nm}\right)$. The UV light was generated from a $100 \mathrm{~W}$ mercury lamp $(365 \mathrm{~nm}$, USH-1030L, USHIO Inc.), focused through a 40x objective and the DAPI-5060COMF-ZERO filter set (Semrock).

To quantitatively measure the release of FITC-dextran and Nile Red, we collected microfluidic droplets for $6 \mathrm{~h}$ in a $2 \mathrm{~mL}$ vial. After overnight evaporation of the oil and aqueous phases, the prepared microcapsules within the vial were hydrated in water $(800 \mu \mathrm{L})$ to measure the fluorescence spectrum of any released cargo. The 
photochemical disassembly of the microcapsules was triggered by exposure to a UV light source (LZC-ORG photoreactor with 377/50 nm filter).

\section{ASSOCIATED CONTENT}

\section{S Supporting Information}

The Supporting Information is available free of charge on the ACS Publications website at DOI: 10.1021/acsami.6b00661.

${ }^{1} \mathrm{H}$ NMR spectrum of polymers $\mathbf{P 1}$ and P2, the characterizations of $\mathbf{P} \mathbf{1}$ micelles, the fluorescence images of microcapsules, and the fluorescence spectra of FITCdextran and Nile Red. (PDF)

\section{AUTHOR INFORMATION}

\section{Corresponding Authors}

*E-mail: ca26@cam.ac.uk.

*E-mail: oas23@cam.ac.uk.

\section{Author Contributions}

${ }^{\ddagger}$ Z.Y. and Y.Z. contributed equally to this work.

Notes

The authors declare no competing financial interest.

\section{ACKNOWLEDGMENTS}

This work was supported by the Engineering and Physical Sciences Research Council, Institutional Sponsorship 2012University of Cambridge EP/K503496/1 and the Translational Grant EP/H046593/1; Y.Z. and R.P. were also funded from the European Research Council Starting Investigator grant ASPiRe (No. 240629) and the Isaac Newton Trust research grant No. 13.7(c). R.C. received funding from the European Research Council under the European Union's Seventh Framework Programme (FP/2007-2013)/ERC Proof of Concept Grant Agreement n. 297504; Y. Lan is supported by the CSC Cambridge Scholarship. Z.Y. thanks C. Hu for helpful discussions. Additional data related to this publication is available at the University of Cambridge data repository (https://www.repository.cam.ac.uk/handle/1810/248506).

\section{REFERENCES}

(1) Lehn, J.-M. Toward Complex Matter: Supramolecular Chemistry and Self-organization. Proc. Natl. Acad. Sci. U. S. A. 2002, 99, 47634768.

(2) Zhang, S. Fabrication of Novel Biomaterials through Molecular Self-assembly. Nat. Biotechnol. 2003, 21, 1171-1178.

(3) Pouget, E.; Dujardin, E.; Cavalier, A.; Moreac, A.; Valery, C.; Marchi-Artzner, V.; Weiss, T.; Renault, A.; Paternostre, M.; Artzner, F. Hierarchical Architectures by Synergy Between Dynamical Template Self-assembly and Biomineralization. Nat. Mater. 2007, 6, 434-439.

(4) Groschel, A. H.; Walther, A.; Lobling, T. I.; Schacher, F. H.; Schmalz, H.; Müller, A. H. E. Guided Hierarchical Co-assembly of Soft Patchy Nanoparticles. Nature 2013, 503, 247-251.

(5) Li, Z.; Ma, J.; Lee, N. S.; Wooley, K. L. Dynamic Cylindrical Assembly of Triblock Copolymers by a Hierarchical Process of Covalent and Supramolecular Interactions. J. Am. Chem. Soc. 2011, 133, 1228-1231.

(6) Yao, H.-B.; Fang, H.-Y.; Wang, X.-H.; Yu, S.-H. Hierarchical Assembly of Micro-/nano-Building Blocks: Bio-inspired Rigid Structural Functional Materials. Chem. Soc. Rev. 2011, 40, 3764-3785.

(7) Zhu, J.; Zhang, S.; Zhang, F.; Wooley, K. L.; Pochan, D. J. Hierarchical Assembly of Complex Block Copolymer Nanoparticles into Multicompartment Superstructures Through Tunable Interparticle Associations. Adv. Funct. Mater. 2013, 23, 1767-1773.

(8) Schreiber, R.; Do, J.; Roller, E.-M.; Zhang, T.; Schuller, V. J.; Nickels, P. C.; Feldmann, J.; Liedl, T. Hierarchical Assembly of Metal
Nanoparticles, Quantum Dots and Organic Dyes using DNA Origami Scaffolds. Nat. Nanotechnol. 2014, 9, 74-78.

(9) Yu, Z.-Y.; Wang, C.-F.; Chen, S. Fabrication of Quantum Dotbased Photonic Materials from Small to Large via Interfacial Selfassembly. J. Mater. Chem. 2011, 21, 8496-8501.

(10) Kita-Tokarczyk, K.; Grumelard, J.; Haefele, T.; Meier, W. Block Copolymer Vesicles-using Concepts from Polymer Chemistry to Mimic Biomembranes. Polymer 2005, 46, 3540-3563.

(11) Jin, H.; Huang, W.; Zhu, X.; Zhou, Y.; Yan, D. Biocompatible or Biodegradable Hyperbranched Polymers: from Self-assembly to Cytomimetic Applications. Chem. Soc. Rev. 2012, 41, 5986-5997.

(12) Marguet, M.; Bonduelle, C.; Lecommandoux, S. Multicompartmentalized Polymeric Systems: towards Biomimetic Cellular Structure and Function. Chem. Soc. Rev. 2013, 42, 512-529.

(13) Kim, K. T.; Zhu, J.; Meeuwissen, S. A.; Cornelissen, J. J. L. M.; Pochan, D. J.; Nolte, R. J. M.; van Hest, J. C. M. Polymersome Stomatocytes: Controlled Shape Transformation in Polymer Vesicles. J. Am. Chem. Soc. 2010, 132, 12522-12524.

(14) Kim, K. T.; Zhu, J.; Meeuwissen, S. A.; Cornelissen, J. J. L. M.; Pochan, D. J.; Nolte, R. J. M.; van Hest, J. C. M. Polymersome Stomatocytes: Controlled Shape Transformation in Polymer Vesicles. J. Am. Chem. Soc. 2010, 132, 12522-12524.

(15) Yan, Q.; Zhou, R.; Fu, C.; Zhang, H.; Yin, Y.; Yuan, J. $\mathrm{CO}_{2-}^{-}$ Responsive Polymeric Vesicles that Breathe. Angew. Chem. 2011, 123, 5025-5029.

(16) Kumar, M.; Habel, J. E. O.; Shen, Y.-X.; Meier, W. P.; Walz, T. High-Density Reconstitution of Functional Water Channels into Vesicular and Planar Block Copolymer Membranes. J. Am. Chem. Soc. 2012, 134, 18631-18637.

(17) Stuart, M. A. C.; Huck, W. T. S.; Genzer, J.; Muller, M.; Ober, C.; Stamm, M.; Sukhorukov, G. B.; Szleifer, I.; Tsukruk, V. V.; Urban, M.; Winnik, F.; Zauscher, S.; Luzinov, I.; Minko, S. Emerging Applications of Stimuli-Responsive Polymer Materials. Nat. Mater. 2010, 9, 101-113.

(18) Cabral, H.; Matsumoto, Y.; Mizuno, K.; Chen, Q.; Murakami, M.; Kimura, M.; Terada, Y.; Kano, M. R.; Miyazono, K.; Uesaka, M.; Nishiyama, N.; Kataoka, K. Accumulation of Sub-100 nm Polymeric Micelles in Poorly Permeable Tumours Depends on Size. Nat. Nanotechnol. 2011, 6, 815-823.

(19) Orilall, M. C.; Wiesner, U. Block Copolymer based Composition and Morphology Control in Nanostructured Hybrid Materials for Energy Conversion and Storage: Solar cells, Batteries, and Fuel cells. Chem. Soc. Rev. 2011, 40, 520-535.

(20) Siegwart, D. J.; Oh, J. K.; Matyjaszewski, K. ATRP in the Design of Functional Materials for Biomedical Applications. Prog. Polym. Sci. 2012, 37, 18-37.

(21) Kelley, E. G.; Albert, J. N. L.; Sullivan, M. O.; Epps, T. H., III. Stimuli-responsive Copolymer Solution and Surface Assemblies for Biomedical Applications. Chem. Soc. Rev. 2013, 42, 7057-7071.

(22) Fang, B.; Walther, A.; Wolf, A.; Xu, Y.; Yuan, J.; Müller, A. H. E. Undulated Multicompartment Cylinders by the Controlled and Directed Stacking of Polymer Micelles with a Compartmentalized Corona. Angew. Chem., Int. Ed. 2009, 48, 2877-2880.

(23) Gröschel, A. H.; Walther, A.; Löbling, T. I.; Schmelz, J.; Hanisch, A.; Schmalz, H.; Müller, A. H. E. Facile, Solution-Based Synthesis of Soft, Nanoscale Janus Particles with Tunable Janus Balance. J. Am. Chem. Soc. 2012, 134, 13850-13860.

(24) Warren, N. J.; Mykhaylyk, O. O.; Ryan, A. J.; Williams, M.; Doussineau, T.; Dugourd, P.; Antoine, R.; Portale, G.; Armes, S. P. Testing the Vesicular Morphology to Destruction: Birth and Death of Diblock Copolymer Vesicles Prepared via Polymerization-Induced Self-Assembly. J. Am. Chem. Soc. 2015, 137, 1929-1937.

(25) Moulton, B.; Zaworotko, M. J. From Molecules to Crystal Engineering: Supramolecular Isomerism and Polymorphism in Network Solids. Chem. Rev. 2001, 101, 1629-1658.

(26) Dsouza, R. N.; Pischel, U.; Nau, W. M. Fluorescent Dyes and Their Supramolecular Host/Guest Complexes with Macrocycles in Aqueous Solution. Chem. Rev. 2011, 111, 7941-7980. 
(27) Zhang, Z.; Luo, Y.; Chen, J.; Dong, S.; Yu, Y.; Ma, Z.; Huang, F. Formation of Linear Supramolecular Polymers That Is Driven by C$\mathrm{H} \cdots \pi$ Interactions in Solution and in the Solid State. Angew. Chem., Int. Ed. 2011, 50, 1397-1401.

(28) Dong, S.; Zheng, B.; Wang, F.; Huang, F. Supramolecular Polymers Constructed from Macrocycle-Based Host-Guest Molecular Recognition Motifs. Acc. Chem. Res. 2014, 47, 1982-1994.

(29) Wei, P.; Cook, T. R.; Yan, X.; Huang, F.; Stang, P. J. A Discrete Amphiphilic Organoplatinum(II) Metallacycle with Tunable Lower Critical Solution Temperature Behavior. J. Am. Chem. Soc. 2014, 136, 15497-15500.

(30) Zhou, Y.; Wang, D.; Huang, S.; Auernhammer, G.; He, Y.; Butt, H.-J.; Wu, S. Reversible Janus Particle Assembly via Responsive Hostguest Interactions. Chem. Commun. 2015, 51, 2725-2727.

(31) Krieg, E.; Weissman, H.; Shirman, E.; Shimoni, E.; Rybtchinski, B. A Recyclable Supramolecular Membrane for Size-selective Separation of Nanoparticles. Nat. Nanotechnol. 2011, 6, 141-146.

(32) Kurihara, K.; Tamura, M.; Shohda, K.-i.; Toyota, T.; Suzuki, K.; Sugawara, T. Self-Reproduction of Supramolecular Giant Vesicles Combined with the Amplification of Encapsulated DNA. Nat. Chem. 2011, 3, 775-781.

(33) Aida, T.; Meijer, E. W.; Stupp, S. I. Functional Supramolecular Polymers. Science 2012, 335, 813-817.

(34) Schmidt, B. V. K. J.; Hetzer, M.; Ritter, H.; Barner-Kowollik, C. UV Light and Temperature Responsive Supramolecular ABA Triblock Copolymers via Reversible Cyclodextrin Complexation. Macromolecules 2013, 46, 1054-1065.

(35) Chen, P.; Luo, Z.; Güven, S.; Tasoglu, S.; Ganesan, A. V.; Weng, A.; Demirci, U. Microscale Assembly Directed by Liquid-Based Template. Adv. Mater. 2014, 26, 5936-5941.

(36) Kim, S.-Y.; Jung, I.-S.; Lee, E.; Kim, J.; Sakamoto, S.; Yamaguchi, K.; Kim, K. Macrocycles within Macrocycles: Cyclen, Cyclam, and Their Transition Metal Complexes Encapsulated in Cucurbit[8]uril. Angew. Chem. 2001, 113, 2177-2179.

(37) Moon, K.; Grindstaff, J.; Sobransingh, D.; Kaifer, A. E. Cucurbit[8]uril-Mediated Redox-Controlled Self-Assembly of Viologen-Containing Dendrimers. Angew. Chem., Int. Ed. 2004, 43, 54965499.

(38) Rauwald, U.; Scherman, O. A. Supramolecular Block Copolymers with Cucurbit[8]uril in Water. Angew. Chem., Int. Ed. 2008, 47, 3950-3953.

(39) Song, Q.; Li, F.; Wang, Z.; Zhang, X. A supramolecular strategy for tuning the energy level of naphthalenediimide: Promoted formation of radical anions with extraordinary stability. Chem. Sci. 2015, 6, 3342-3346.

(40) Appel, E. A.; Biedermann, F.; Rauwald, U.; Jones, S. T.; Zayed, J. M.; Scherman, O. A. Supramolecular Cross-Linked Networks via Host-Guest Complexation with Cucurbit[8]uril. J. Am. Chem. Soc. 2010, 132, 14251-14260.

(41) Lan, Y.; Wu, Y.; Karas, A.; Scherman, O. A. Photoresponsive Hybrid Raspberry-Like Colloids Based on Cucurbit[8]uril HostGuest Interactions. Angew. Chem., Int. Ed. 2014, 53, 2166-2169.

(42) Liu, Y.; Yu, Y.; Gao, J.; Wang, Z.; Zhang, X. Water-Soluble Supramolecular Polymerization Driven by Multiple Host-Stabilized Charge-Transfer Interactions. Angew. Chem. 2010, 122, 6726-6729.

(43) Rauwald, U.; Barrio, J. d.; Loh, X. J.; Scherman, O. A. "Ondemand" Control of Thermoresponsive Properties of Poly(Nisopropylacrylamide) with Cucurbit[8]uril Host-guest Complexes. Chem. Commun. 2011, 47, 6000-6002.

(44) Liu, Y.; Wang, Z.; Zhang, X. Characterization of Supramolecular Polymers. Chem. Soc. Rev. 2012, 41, 5922-5932.

(45) Zhang, J.; Coulston, R. J.; Jones, S. T.; Geng, J.; Scherman, O. A.; Abell, C. One-Step Fabrication of Supramolecular Microcapsules from Microfluidic Droplets. Science 2012, 335, 690-694.

(46) Stephenson, G.; Parker, R. M.; Lan, Y.; Yu, Z.-Y.; Scherman, O. A.; Abell, C. Supramolecular Colloidosomes: Fabrication, Characterisation and Triggered Release of Cargo. Chem. Commun. 2014, 50, 7048-7051.
(47) Zheng, Y.; Yu, Z.-Y.; Parker, R. M.; Wu, Y.; Abell, C.; Scherman, O. A. Interfacial Assembly of Dendritic Microcapsules with HostGuest Chemistry. Nat. Commun. 2014, 5, 5772.

(48) Schacher, F. H.; Rupar, P. A.; Manners, I. Functional Block Copolymers: Nanostructured Materials with Emerging Applications. Angew. Chem., Int. Ed. 2012, 51 (32), 7898-7921.

(49) Ji, X.; Dong, S.; Wei, P.; Xia, D.; Huang, F. A Novel Diblock Copolymer with a Supramolecular Polymer Block and a Traditional Polymer Block: Preparation, Controllable Self-Assembly in Water, and Application in Controlled Release. Adv. Mater. 2013, 25 (40), 57255729.

(50) Teh, S.-Y.; Lin, R.; Hung, L.-H.; Lee, A. P. Droplet Microfluidics. Lab Chip 2008, 8, 198-220.

(51) Casadevall i Solvas, X.; deMello, A. Droplet Microfluidics: Recent Developments and Future Applications. Chem. Commun. 2011, 47, 1936-1942.

(52) Yu, Z.-Y.; Wang, C.-F.; Ling, L.; Chen, L.; Chen, S. Triphase Microfluidic-Directed Self-Assembly: Anisotropic Colloidal Photonic Crystal Supraparticles and Multicolor Patterns Made Easy. Angew. Chem., Int. Ed. 2012, 51, 2375-2378.

(53) Parker, R. M.; Zhang, J.; Zheng, Y.; Coulston, R. J.; Smith, C. A.; Salmon, A. R.; Yu, Z.-Y.; Scherman, O. A.; Abell, C. Electrostatically Directed Self-Assembly of Ultrathin Supramolecular Polymer Microcapsules. Adv. Funct. Mater. 2015, 25, 4091-4100.

(54) Yu, Z.-Y.; Zhang, J.; Coulston, R.; Parker, R. M.; Biedermann, F.; Liu, X.; Scherman, O. A.; Abell, C. Supramolecular Hydrogel Microcapsules via Cucurbit[8]uril Host-guest Interactions with Triggered and UV-Controlled Molecular Permeability. Chem. Sci. 2015, 6, 4929-4933.

(55) Chen, P. W.; Cadisch, G.; Studart, A. R. Encapsulation of Aliphatic Amines Using Microfluidics. Langmuir 2014, 30, 2346-2350.

(56) Ge, X.-H.; Huang, J.-P.; Xu, J.-H.; Luo, G.-S. Controlled Stimulation-Burst Targeted Release by Smart Decentered Core-shell Microcapsules in Gravity and Magnetic Field. Lab Chip 2014, 14, 4451-4454.

(57) Wei, J.; Ju, X.-J.; Zou, X.-Y.; Xie, R.; Wang, W.; Liu, Y.-M.; Chu, L.-Y. Multi-Stimuli-Responsive Microcapsules for Adjustable Controlled-Release. Adv. Funct. Mater. 2014, 24, 3312-3323.

(58) Zieringer, M. A.; Carroll, N. J.; Abbaspourrad, A.; Koehler, S. A.; Weitz, D. A. Microcapsules for Enhanced Cargo Retention and Diversity. Small 2015, 11, 2903-2909.

(59) Chen, P. W.; Erb, R. M.; Studart, A. R. Designer Polymer-Based Microcapsules Made Using Microfluidics. Langmuir 2012, 28, 144152

(60) Pessi, J.; Santos, H. A.; Miroshnyk, I.; Yliruusi, J.; Weitz, D. A.; Mirza, S. Microfluidics-Assisted Engineering of Polymeric Microcapsules with High Encapsulation Efficiency for Protein Drug Delivery. Int. J. Pharm. 2014, 472, 82-87.

(61) Duncanson, W. J.; Lin, T.; Abate, A. R.; Seiffert, S.; Shah, R. K.; Weitz, D. A. Microfluidic Synthesis of Advanced Microparticles for Encapsulation and Controlled Release. Lab Chip 2012, 12, 21352145.

(62) Riahi, R.; Tamayol, A.; Shaegh, S. A. M.; Ghaemmaghami, A. M.; Dokmeci, M. R.; Khademhosseini, A. Microfluidics for Advanced Drug Delivery Systems. Curr. Opin. Chem. Eng. 2015, 7, 101-112.

(63) Wang, W.; Xie, R.; Ju, X.-J.; Luo, T.; Liu, L.; Weitz, D. A.; Chu, L.-Y. Controllable Microfluidic Production of Multicomponent Multiple Emulsions. Lab Chip 2011, 11, 1587-1592.

(64) Deye, J. F.; Berger, T. A.; Anderson, A. G. Nile Red as a Solvatochromic Dye for Measuring Solvent Strength in Normal Liquids and Mixtures of Normal Liquids with Supercritical and Near Critical Fluids. Anal. Chem. 1990, 62, 615-622.

(65) Kurniasih, I. N.; Liang, H.; Mohr, P. C.; Khot, G.; Rabe, J. P.; Mohr, A. Nile Red Dye in Aqueous Surfactant and Micellar Solution. Langmuir 2015, 31, 2639-2648.

(66) Jaiswal, J. K.; Chakrabarti, S.; Andrews, N. W.; Simon, S. M. Synaptotagmin VII Restricts Fusion Pore Expansion during Lysosomal Exocytosis. PLoS Biol. 2004, 2, 1224-1232. 
(67) del Barrio, J.; Horton, P. N.; Lairez, D.; Lloyd, G. O.; Toprakcioglu, C.; Scherman, O. A. Photocontrol over Cucurbit[8]uril Complexes: Stoichiometry and Supramolecular Polymers. J. Am. Chem. Soc. 2013, 135, 11760-11763.

(68) Day, A.; Arnold, A. P.; Blanch, R. J.; Snushall, B. Controlling Factors in the Synthesis of Cucurbituril and Its Homologues. J. Org. Chem. 2001, 66 (24), 8094-8100.

(69) Kim, J.; Jung, I.-S.; Kim, S.-Y.; Lee, E.; Kang, J.-K.; Sakamoto, S.; Yamaguchi, K.; Kim, K. New Cucurbituril Homologues: Syntheses, Isolation, Characterization, and X-ray Crystal Structures of Cucurbit[n] uril (n = 5, 7, and 8). J. Am. Chem. Soc. 2000, 122, 540-541. 